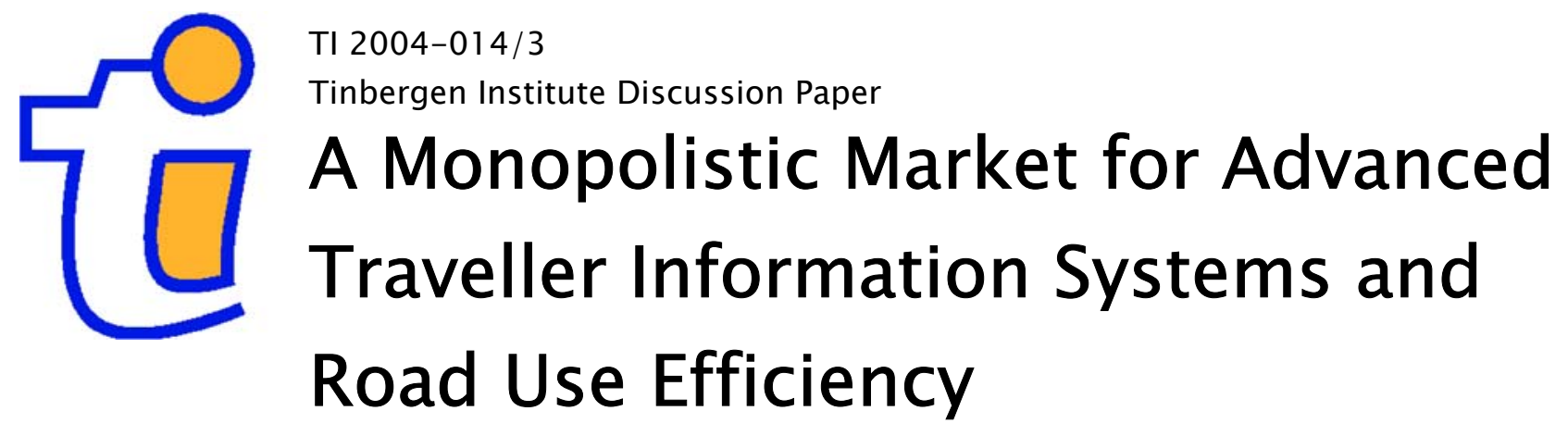

Rong Zhang

Erik T. Verhoef

Department of Spatial Economics, Faculty of Economics and Business Administration, Vrije Universiteit Amsterdam, and Tinbergen Institute. 


\section{Tinbergen Institute}

The Tinbergen Institute is the institute for economic research of the Erasmus Universiteit Rotterdam, Universiteit van Amsterdam, and Vrije Universiteit Amsterdam.

Tinbergen Institute Amsterdam

Roetersstraat 31

1018 WB Amsterdam

The Netherlands

Tel.: $\quad+31(0) 205513500$

Fax: $\quad+31(0) 205513555$

Tinbergen Institute Rotterdam

Burg. Oudlaan 50

3062 PA Rotterdam

The Netherlands

Tel.: $\quad+31(0) 104088900$

Fax: $\quad+31(0) 104089031$

Please send questions and/or remarks of nonscientific nature to driessen@tinbergen.nl.

Most TI discussion papers can be downloaded at http://www.tinbergen.nl. 


\title{
A Monopolistic Market for Advanced Traveller Information Systems and Road Use Efficiency
}

\author{
Rong Zhang* and Erik T. Verhoef \\ Department of Spatial Economics, Free University Amsterdam \\ De Boelelaan 1105, 1081 HV Amsterdam, The Netherlands
}

\begin{abstract}
This study presents a model for exploring the interactions between a transport market and a monopolistic market for advanced traveller information systems (ATIS). We examine the properties of the information market, and in particular, the relationship between monopoly information pricing and road system efficiency. From model experiments, we find that increasing information penetration will lead to negative external effects for the already informed drivers. Furthermore, monopoly information pricing is not the most attractive option from a system efficiency viewpoint, because of the relatively high price charged by the monopolist. Third, a user or a producer subsidy on the information market can help realise a second-best optimum of road use. Finally, relatively low uncertainty on the road and high information costs limit the monopolist's profit on the information market, as well as relative system efficiency. While relatively inelastic demand for mobility negatively affects the monopolist's profit, the relative social benefits from private information peak at intermediate demand elasticities.
\end{abstract}

Topic area: Telecommunications and Advanced Information Systems

Keywords: Advanced Traveller Information Systems; Willingness to pay for information; Monopoly information pricing; Road use efficiency; Second-best optimum

\section{Introduction}

The development in the literature regarding the impacts of (ATIS) on road transport has been significant in recent years. ${ }^{1}$ It has been shown in various simulation works that drivers equipped with ATIS benefit to differing degrees, with estimates varying from 7 to 20 percent of travel time savings, depending on the road network under consideration. System performance also improves, in terms of overall time savings in the network, by 1 up to 55 percent, at various levels of market penetration. ${ }^{2}$ Over the long run, such systems may reduce the need to construct additional highway infrastructure. On the other hand, they may induce additional demand. With ATIS, each traveller individually, and the road network as a whole, could be made more productive (Levinson, 2003).

Over the next years, drivers may be expected to become more informed with the advent and deployment of ATIS, although issues such as information quality and costs may remain a main concern. In field operational tests of ATIS in the USA, results have shown that users are generally appreciative of traffic information as long, not surprisingly, as they perceive the information to be accurate, timely and inexpensive. However, some technical problems related to information quality of en-route systems are yet to be solved. There

\footnotetext{
* Corresponding author. Tel: +31 20 4446142; Fax: +31 20 4446004; E-mail: rzhang@feweb.vu.nl (R. Zhang).

${ }^{1}$ The ATIS are called motorist information systems in Emmerink (1998).

${ }^{2}$ See Levinson (2003), where benefits to equipped drivers and improvement in system performance in the models are summarized.
} 
appears to be a certain willingness to pay for some travel information systems; however, market penetration by 'high-end' systems is believed to be limited until costs decrease (Noonan and Shearer, 1998). Currently the public sector is often dominating information supply, but we might also expect private initiatives. A possible advantage might be that private firms would have incentives to improve the quality and costs of information through, for example, adopting new communications technologies on the one hand, and pursuing economies of scale on the other.

Following Emmerink's approach (1998), the present study aims at developing a model system in which a transport market and a market for ATIS interact. While Emmerink has introduced endogenously determined demand for information on the road, the present paper proceeds by explicitly constructing a monopolist market for information. The inherent mutual interactions between road use and benefits from traveller information are accounted for in our model. Based on this, we are able to analyse the effects of monopoly information pricing on road system efficiency. In addition, the present paper differs from Emmerink's work by including an alternative, more systematic decomposition of welfare effects of information. In this way, the positive and negative external effects of information and congestion externalities on road users' information benefits are identified, which is important for assessing the efficiency impacts of information provision.

The organisation of the paper is as follows. Section 2 describes the model, where important assumptions are made, and the model and its equilibrium conditions are specified. Section 3 addresses some conceptual fundamentals, and the simulation results with respect to the four aspects mentioned above. Section 4 concludes.

\section{The model}

\subsection{Model assumptions}

The model system integrates a road transport market and an information market. The transport market is presented in its simplest possible form - one link, while allowing for static congestion. Assuming one driver per vehicle, the inverse demand for mobility, reflecting travellers' private benefits from the trip, is represented by a downward-sloping curve, $D(N)$, where $N$ denotes the number of road users or trips. On the supply side, the marginal private cost function, equal to the average social cost function (upward-sloping to reflect congestion costs), is given by $C^{0}(N)$ or $C^{1}(N)$, depending on the state of the road (indicated by the superscript). State 1 refers to low road capacity, while state 0 stands for high capacity. The two states occur with probabilities $p$ and 1-p respectively. Both travel cost functions are increasing in $N$. In addition, it is assumed that the travel costs itself and the rate at which travel costs increase in $N$ are higher in state 1. That is, the relationships that

$$
C^{l}(N)>C^{0}(N) \text { and } \mathrm{d} C^{l}(N) / \mathrm{d} N>\mathrm{d} C^{0}(N) / \mathrm{d} N
$$

hold for all $N$. The low road capacity in state 1 is caused by random (unpredictable) incidents such as traffic accidents, sudden lane closures, etc. By this means, stochasticity is introduced into the road, which renders it possible for analysing the impact of information on the transport market.

In the model, we assume that all drivers are familiar with average traffic conditions. Without information, we assume that drivers' decision-making is based on unbiased, expected traffic conditions (there is no risk aversion). When these assumptions are translated into the model, the situation is as follows. Uninformed drivers use the probabilities, and the correctly predicted equilibrium average costs in both states, to 
determine the expected costs on the road. When drivers are armed with real-time pre-trip information from ATIS, they will make trip decisions according to actual traffic situations, since they know which state is prevailing. ${ }^{3}$ Consequently, an informed driver will use the road only if his private benefit is at least equal to the actual private costs for the prevailing state. An uninformed driver uses the network if private benefit does not fall short of expected private costs. Both groups of drivers can be characterized as utility maximizers.

As will be seen in next section, when a logical assumption is made that drivers benefiting directly from information are indeed willing to pay for it, demand for information can be derived from the conditions on the transport market. With the supply of information (equipment) assumed coming from a monopolistic, profit-maximizing firm, a market for information is then formed. The supplier starts to provide information to the road users once the information equipment is sold. So the full product has two components: the 'hardware', the equipment, and the 'software', the pre-trip information. ${ }^{4}$

\section{Figure 1 Conceptual framework of the model system}

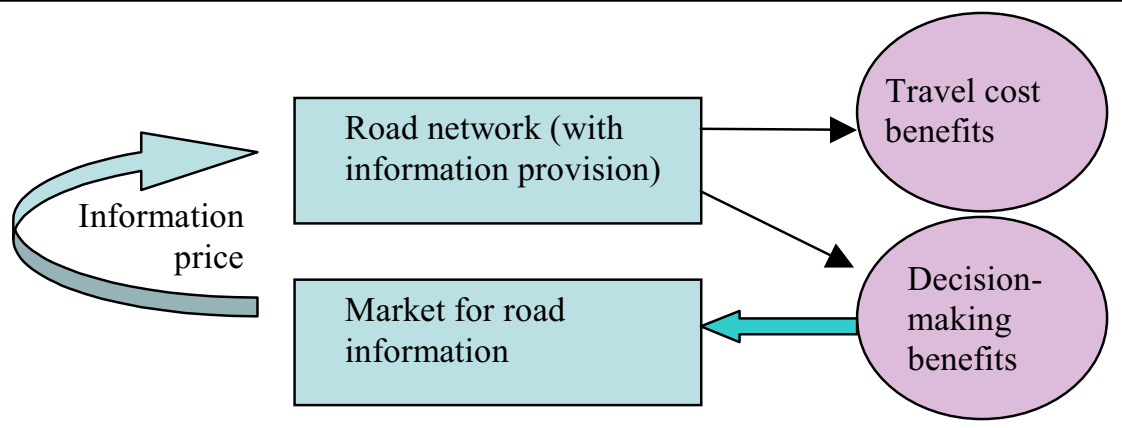

Figure 1 presents the interdependence of the transport and information market. Benefits from information are divided into external and internal benefits. While external benefits are in nature independent of whether or not the driver himself is informed, internal benefits are used to derive the demand side of the information market. After price of information has been formed as a result of the interactions between information buyers and the information provider, there will be a feedback to the transport market, which in turn affects system performance, and hence the value of information. In this way, the two markets are mutually dependent.

\subsection{Model formulation}

\subsubsection{Exogenous information on the road}

To introduce our full model, we first present two models that reflect the benchmark cases of no information (Model N) and free but perfect information (Model P) on the road. This is helpful in identifying the factors underlying the willingness to pay for information. Model $\mathrm{P}$ is equivalent to the situation of $100 \%$ market penetration of information. Using the behavioural principles explained in the previous section, equilibrium conditions of the two benchmark models can be expressed as

\footnotetext{
${ }^{3}$ One can think of devices such as digital watches, in-vehicle devices and PCs, used to supply pre-trip information in the Field Operational Tests in the USA (Noonan and Shearer, 1998). These devices can provide traffic information, incident reports, rideshare matching and transit schedules. For our model incident reports are most relevant.

${ }^{4}$ From here onwards, information and the equipment will be used interchangeably.
} 
Model $\mathrm{N} \quad D\left(N_{N}\right)=(1-p) C^{0}\left(N_{N}\right)+p C^{1}\left(N_{N}\right)$

Model P $\quad D\left(N_{P}^{0}\right)=C^{0}\left(N_{P}^{0}\right)$

$D\left(N_{P}^{1}\right)=C^{1}\left(N_{P}^{1}\right)$,

where $N_{N}, N_{P}^{0}$ and $N_{P}^{1}$ refer to the equilibrium numbers of road users in Model N, and in Model $\mathrm{P}$ in states 0 and 1 , respectively. ${ }^{5}$ They thus identify the marginal drivers, who are indifferent between using the road or not in the three equilibria. Eq.(1) states that for the marginal driver $N_{N}$, his private benefit is equal to his expected travel costs. In Eq.(2), the marginal drivers $N_{P}^{0}$ and $N_{P}^{1}$ equate their private benefit with the actual travel costs in states 0 and 1 under perfect information.

In Emmerink (1998, Chap 5), it is proven that, assuming a linear function system for the transport market, expected road usage increases while expected travel costs decrease in Model P relative to Model N. As a result, an expected welfare gain arises in the road system due to information (a strict Pareto improvement). ${ }^{67}$

The left panel in Figure 2 illustrates the welfare gains/losses of the drivers in Model $\mathrm{P}$ relative to Model $\mathrm{N}^{8}{ }^{8}$ In the figure, $D$ gives the inverse demand function for road use. The horizontal lines, denoted by $C$ with differing sub- and superscripts, represent equilibrium cost levels in the models. They should therefore not be mistaken to represent the (upward-sloping) travel cost functions. The comparison between the models in terms of equilibrium road usage and levels of travel costs are given below.

$$
\begin{aligned}
& C_{N}^{0} \leq C_{P}^{0} \leq C_{P} \leq C_{N} \leq C_{P}^{1} \leq C_{N}^{1} \\
& N_{P}^{1} \leq N_{N} \leq N_{P}^{0},
\end{aligned}
$$

where $C_{N}=(1-p) C_{N}^{0}+p C_{N}^{1}$ denotes expected travel costs in Model $\mathrm{N}$, and $C_{P}$ (not depicted in Figure 2), those in Model P. With information, more drivers (by the amount $N_{P}^{0}-N_{N}$ ) will use the road in state 0 when being informed that high road capacity is prevailing. In state 1 , drivers between $N_{N}$ and $N_{P}^{1}$ refrain from travelling, because actual travel costs would exceed their willingness to pay for mobility. Consequently, drivers between $N_{P}^{1}$ and $N_{P}^{0}$ will change their behaviour when being informed, which in turn causes welfare changes in the road system.

The total welfare gain due to information comprises two kinds of benefits: travel cost benefits and decision-making benefits. ${ }^{9}$ Travel cost benefits exclusively result from

\footnotetext{
${ }^{5}$ For simplicity, only expressions for the marginal drivers in the equilibria are presented in Model $\mathrm{N}$ and $\mathrm{P}$, which, in addition, requires the assumption of 'group regularity'. This assumption says that in each possible state, at least one driver from the specified group is using the road. See Emmerink (1998, Chapters 5 and 6) for more on this.

${ }^{6}$ In one particular state, net welfare effects might be negative, as calculated by the authors using numerical parameter values assigned in Emmerink (1998, Chap 5). In Chap 9 of Emmerink (1998), this issue is explicitly discussed.

${ }^{7}$ Emmerink's Chap 5 assumes explicitly two groups of drivers, informed and uninformed ones. The present paper, a continuation of his Chap 7, endogenizes the separation between informed and uninformed drivers. Nevertheless, the conclusions in Chap 5 apply to the present model.

${ }^{8}$ Figure 2 is a slightly revised version of Figure 7.1 in Emmerink (1998, Chap 7).

${ }^{9}$ These are called external benefits and internal decision-making benefits respectively in Chap 6, Emmerink (1998). Note that in Appendix A.1, it can be seen that both kinds of benefits result from the change in actual travel costs in each state.
} 
decreased congestion externalities. In state 0 , the fact that more drivers use the road will cause a higher travel cost, relative to the no information situation $\left(C_{P}^{0}>C_{N}^{0}\right)$, leading to an increased congestion externality. Therefore, all drivers between 0 and $N_{N}$ will suffer in this state, with the amount equalling the lower shaded rectangle $T T C L^{0}$ (total travel cost losses in state 0$)$ in the left panel of Figure 2. However, in state 1, the opposite occurs $\left(C_{N}^{1}<\right.$ $C_{P}^{1}$ ), resulting in a decreased congestion externality. The same group of drivers will gain in this state, as represented by the upper shaded rectangle $T T C S^{l}$ (total travel cost savings in state 1) in the same figure. The expected travel costs will fall as an overall result. This is due to the fact that after being informed, the drivers between $N_{P}^{1}$ and $N_{N}$ will avoid the more heavily congested state 1 , from which the savings in travel costs will more than compensate for the increased costs the drivers between $N_{N}$ and $N_{P}^{0}$ cause in the less congested state 0 .

\section{Figure 2 Decomposition of welfare gain from information}

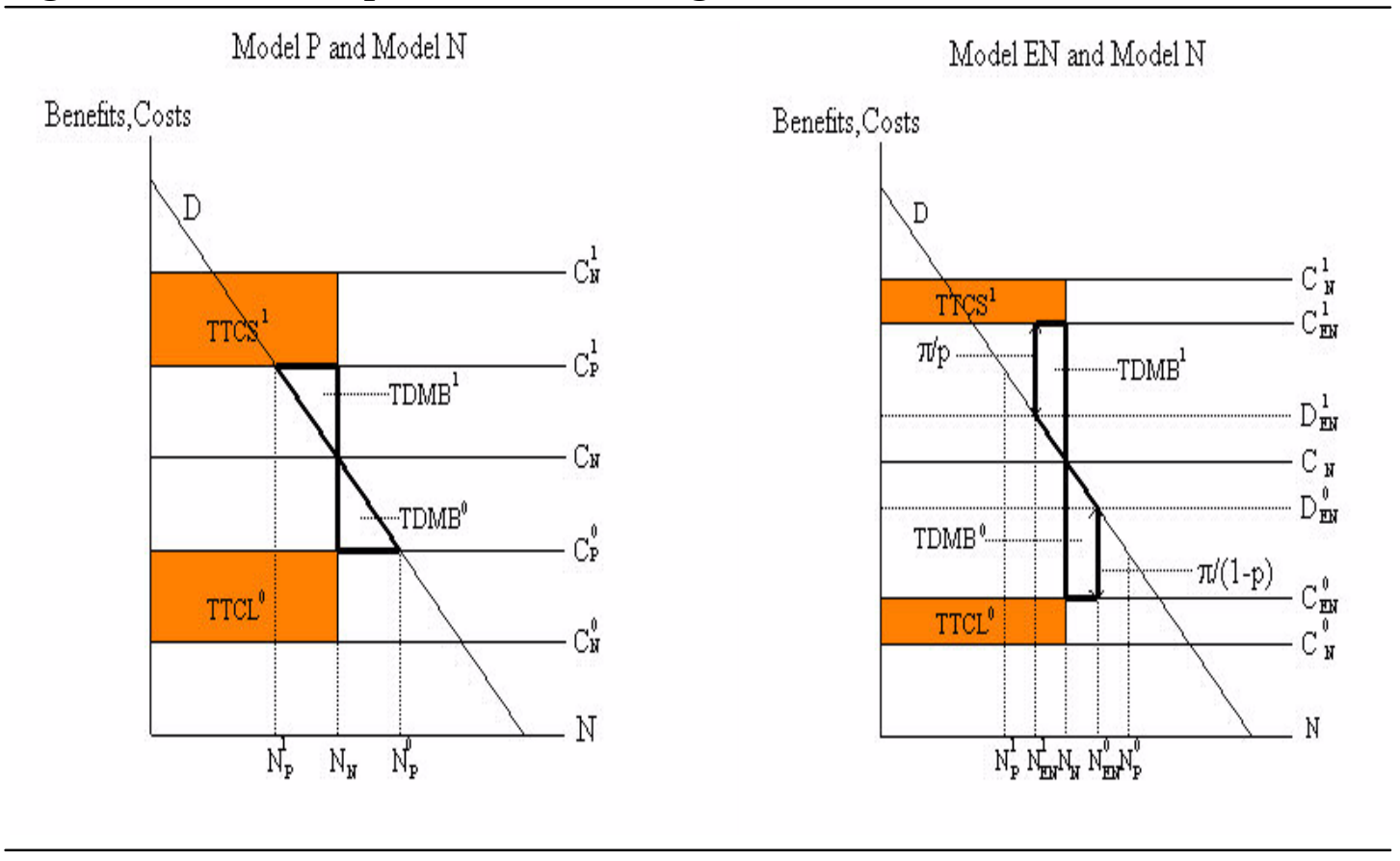

Decision-making benefits, as the name suggests, accrue only to the drivers who change their behaviour - drivers between $N_{P}^{1}$ and $N_{P}^{0}$. In state $0(1)$, the size of the decision-making benefits equals the lower (upper) triangle area highlighted by the heavy solid lines in the left panel of Figure 2, which we denote by $T D M B^{0}\left(T D M B^{1}\right)$ (total decision-making benefits in state $0(1))$. These areas give the net benefits from avoiding the use of the road in state 1 (for drivers between $N_{P}^{1}$ and $N_{N}$ ), or from using it in state 0 (for drivers between $N_{N}$ and $N_{P}^{0}$ ). Note here that each driver perceives his internal decision-making benefits by comparing the situation where he and all other drivers have access to information to the one where he does not but all others do. Total decision-making benefits are the expected value of these two areas. ${ }^{10}$

The difference between travel cost benefits and decision-making benefits lies in the fact that travel cost benefits from information are enjoyed irrespective of whether the

\footnotetext{
${ }^{10}$ For reasons of clarity, the cost levels are equally spaced in Figure 2, although this is not necessarily the case in the numerical cases. As a result, the relative magnitudes of the benefits/losses cannot be visually inferred from the figure.
} 
driver is informed himself. This observation implies that only drivers between $N_{P}^{1}$ and $N_{P}^{0}$ are potentially interested in being informed, since these are the ones that may incur decision-making benefits from the information by changing behaviour. Drivers at the lefthand side of $N_{P}^{1}$ are not interested, since they will use the road regardless the prevailing state, whereas drivers to the right-hand side of $N_{P}^{0}$ are not interested either, since they will never use the road.

\subsubsection{From endogenous demand for information to a market for ATIS}

In this section, we will first derive the demand for information by introducing a model of endogenous information (Model EN). In Model P above, it was assumed that information is an exogenous input into the road system, whereas in the present section, we shift to a situation where a driver's choice of being informed is dependent of the decision-making benefits this driver enjoys. The right panel of Figure 2, which is set up in the same way as the one on the left, depicts the comparison between Model EN and Model N. To facilitate the understanding of Model EN in the expressions (4.a) and (4.b) below, we add the levels of willingness to pay for mobility of the marginal drivers $N_{E N}^{0}$ and $N_{E N}^{1}, D_{E N}^{0}$ and $D_{E N}^{1}$ respectively, to the figure.

Consider driver $N_{E N}^{0}$ in the right-hand panel. In state 0 , if, for this driver, the expected decision-making benefits of having access to information exceed information costs, then the same holds for all drivers between $N_{N}$ and $N_{E N}^{0}$. Similarly, in state 1 , if it is profitable for the driver $N_{E N}^{1}$ to be armed by the information technology, the same will happen to all drivers between $N_{E N}^{1}$ and $N_{N}$. Therefore, if the marginally informed drivers (the drivers who are indifferent between being informed or not) in the two states are $N_{E N}^{0}$ and $N_{E N}^{1}$, then in equilibrium $N_{E N}^{0}-N_{E N}^{1}$ drivers are informed.

Assuming information is supplied at a price $\pi, N_{E N}^{0}$ and $N_{E N}^{1}$ can be determined by equating the expected decision-making benefits for these two drivers with the costs of information. ${ }^{11}$ Eq. (4.a) and (4.b) constitute the two equilibrium conditions of Model EN:

$$
\begin{aligned}
& p\left(C^{1}\left(N_{E N}^{1}\right)-D\left(N_{E N}^{1}\right)\right)=\pi \\
& (1-p)\left(D\left(N_{E N}^{0}\right)-C^{0}\left(N_{E N}^{0}\right)\right)=\pi .
\end{aligned}
$$

Eq.(4.a) states that for the marginally informed driver at $N_{E N}^{1}$, the net expected benefits from being informed, and hence from avoiding road use in state 1 (the LHS), should be equal to the price of information. The costs in state 0 are irrelevant for this individual's willingness to pay for information, as he will use the road in state 0 irrespective of being informed. Eq.(4.b) states that also for the other marginally informed driver at $N_{E N}^{0}$, the expected benefits from being informed, and hence from using the road in state 0 (the LHS), should be equal to price of information. Now the costs in state 1 are irrelevant for his willingness to pay for information, as this user will never use the road in state 1 irrespective of being informed. We emphasize that we have two marginally informed

\footnotetext{
${ }^{11}$ As the model is static in nature, Emmerink (1998) assumes $\pi$ as the daily equivalent of some purchase price (for example leasing, where $\pi$ reflects daily interest and depreciation), or views $\pi$ as the real purchase price and take $D$ and $C$ to be some discounted measure of the future stream of benefits and costs of road usage.
} 
drivers in this model. Driver $N_{E N}^{1}$ is indifferent between being informed and using the road in state 0 only, and being uninformed and always using the road. Driver $N_{E N}^{0}$, in contrast, is indifferent between being informed and using the road in state 0 only, and never using the road.

The resulting network situation can be characterized as follows. First, the informed drivers (those between $N_{E N}^{0}$ and $N_{E N}^{1}$ ) will only use the network when state 0 is prevailing. Then, drivers on the left-hand side of $N_{E N}^{1}$ will not buy the information, because they will always use the road, independent of being informed themselves. Finally, drivers to the right-hand side of $N_{E N}^{0}$ will not buy the information and will never use the road.

The total welfare effects in Model EN relative to Model $\mathrm{N}$ are illustrated in the right panel of Figure 2. These can be interpreted in the same way as those in the left panel. It can be easily seen that Model P corresponds to a limiting case of Model EN where information has a zero price.

The linear demand and cost functions for road use in Emmerink (1998) are adopted here to examine in more detail the properties of information demand in closed-form expressions. The demand for mobility is $D(N)=d-a \cdot N$, characterized by slope $a$ and intercept $d$. The cost functions read $C^{j}(N)=k^{j}+b^{j} \cdot N$ (for state $j=0,1$ ), and consist of a freeflow cost component $k^{j}$ and a congestion cost component that is assumed to be proportional to total road usage, $N^{j}$, with a factor $b^{j}$. All parameters are non-negative. Solving (4.a) and (4.b) yields the equilibrium road usage in state 0 and 1 in terms of the parameters discussed above

$$
\begin{aligned}
& N_{E N}^{0}(\pi)=\frac{d-k^{0}}{a+b^{0}}-\frac{\pi}{(1-p)\left(a+b^{0}\right)}=N_{P^{-}}^{0} \frac{\pi}{(1-p)\left(a+b^{0}\right)} \\
& N_{E N}^{1}(\pi)=\frac{d-k^{l}}{a+b^{l}}+\frac{\pi}{p\left(a+b^{l}\right)}=N_{P}^{l}+\frac{\pi}{p\left(a+b^{l}\right)}
\end{aligned}
$$

where $\pi$ is in the interval $\left[0, \pi_{\max }\right]$ to ensure that $N_{E N}^{1}$ is smaller than $N_{E N}^{0}$ (see (A20)-(A22) in the appendix for the derivation of $\pi_{\max }$ ). Combining (5.a) and (5.b), the total number of informed drivers $N_{E N}^{I n f}$ can be derived as:

$$
N_{E N}^{I n f}(\pi)=N_{E N}^{0}(\pi)-N_{E N}^{1}(\pi)=N_{P}^{0}-N_{P}^{1}-\pi\left(\frac{1}{(1-p)\left(a+b^{0}\right)}+\frac{1}{p\left(a+b^{1}\right)}\right),
$$

where $N_{E N}^{I n f}(\pi)$ refers to information demand as a function of $\pi$. Eq.(6) shows that $N_{E N}^{\operatorname{Inf}}$ is linear and decreasing in $\pi$. At $\pi_{\max }$, demand for information is zero, whereas when $\pi$ is zero, the number of informed drivers is $N_{P}^{0}-N_{\mathrm{P}}^{1}$, which is equivalent to the equilibrium in Model P (we ignore that drivers to the left of $N_{E N}^{1}$ may choose to become informed, too, when $\pi$ falls to zero, without benefiting nor losing from being informed). Thus, we have derived a demand function for information that is entirely based on the parameters of the underlying transport system.

After having derived the demand for ATIS, we now shift to the supply side of the ATIS market, and to the formation of the information price. 
Considering the likely economies of scale in the production of traffic information, we assume a cost function with a fixed cost, $F C$, and constant marginal cost, $\mu{ }^{12}$ The total cost function for supplying information, labelled $T C$, is,

$$
T C=F C+\mu N_{E N}^{I n f}(\pi) .
$$

The decision problem of the monopolist consists of choosing a price $\pi$ so as to maximize his profits, that is, of solving

$$
\operatorname{Max}_{\pi} \pi N_{E N}^{I n f}(\pi)-\left(F C+\mu N_{E N}^{I n f}(\pi)\right)
$$

Suppose the profit-maximizing price is $\pi_{m}$, it must satisfy the first-order condition

$$
N_{E N}^{I n f}\left(\pi_{m}\right)+\left(\pi_{m}-\mu\right) \frac{\mathrm{d} N_{E N}^{I n f}\left(\pi_{m}\right)}{\mathrm{d} \pi}=0
$$

Solving (9) yields

$$
\pi_{m}=\mu+\frac{1}{2}\left(\frac{p(1-p)\left(a+b^{1}\right)\left(a+b^{0}\right)}{a+p b^{1}+(1-p) b^{0}}-\mu\right) .
$$

The second term in (10), the difference between $\pi_{m}$ and $\mu$, is the monopolistic markup.

\section{Welfare effects of private information provision}

In this section, we discuss the welfare effects of monopolistic information provision, using simulation results as an illustration. For the numerical model, we adopt some of the parameter values used by Emmerink (1998, Chap.7), namely, $k^{0}=k^{1}=20, b^{0}=0.015$, $b^{1}=0.04, p=0.25$. To focus on a range of demand elasticities for mobility that are of special interest, however, we change the value of $d$ and $a$ to $d=160$ and $a=0.11$ respectively. Thus, the expected road usage and travel costs in equilibrium in Model $\mathrm{N}$ are 1067 and 42.67, respectively, with the demand elasticity for mobility at this point being -0.36 . We term this point as the reference point and denote it by $\left.\left(N_{N}, C_{N}\right)\right) .{ }^{13}$ It has been found that in Section 3.1 and 3.2, the model results are subject to the changes in the values of $d$ and $a$, although some general conclusions can of course be drawn independent of these parameters.

When being non-zero, $\mu$ is set at 0.5 and $F C$ at 100 . However, to avoid the unnecessary complexity in analysing and interpreting the experimental outcomes related to information benefits, we set $\mu$ and $F C$ equal to zero in the simulations in Section 3.1-3.3 (the analytical solutions of these experiments, in the appendix, allow for non-zero $\mu$ and $F C$ ). In addition, in some sections, we consider an additional regime where congestion externalities are corrected for by optimal road pricing. This facilitates interpretation, as it removes the second-best aspect of information pricing partly correcting for imperfect (i.e. absent congestion pricing. To distinguish this regime from the no pricing case, we add an ' $*$ ' to the notations in the pricing regime.

\footnotetext{
${ }^{12}$ Here we view $\mu$ and $F C$ as the daily equivalent as the production costs of ATIS over the appropriate time horizon. See Footnote 10 for a similar interpretation of information price $\pi$.

${ }^{13}$ In Emmerink (1998), $d=50, a=0.015$. So $\left(N_{N}, C_{N}\right)=37.59,828$ and the corresponding elasticity is -3.03 .
} 


\subsection{Information and congestion externalities}

As has become clear in the previous section, provision of information on the road gives rise to travel cost benefits and decision-making benefits to the drivers. This results from intertwined effects of information supply and road congestion: information alters road usage, which affects all drivers through the congestion externality and its impact on average travel costs. In this section, we refer to this as 'information and congestion externalities'. These information and congestion externalities have important impacts on road users' benefits and system-wide efficiency. A careful consideration of this issue enables us to gain a better understanding of the welfare effects of information pricing, to be studied in Section 3.2. By analysing the externalities, we wish to answer the question: to what extent will (i) the road users, particularly the informed ones; and (ii), the road system as a whole, gain or lose when more drivers get equipped by ATIS? It turns out to be more straightforward to discuss (ii) before (i).

In what follows, we first give a description of some benefit measures that are relevant for our basis of the analysis. Each of these can be defined both from the total and the marginal perspective. With the numbers of road users being $N_{E N}^{0}$ and $N_{E N}^{1}$ in state 0 and 1 respectively (as in Figure 2), these benefit measures are (in total terms) the following: ${ }^{14}$ 1) Expected total decision-making benefits $(T D M B)$, a weighted (by $p$ and 1- $p$ ) average of decision-making benefits as enjoyed by informed drivers in each state: $T D M B=(1-p) \bullet$ $T D M B^{0}+p \bullet T D M B^{l}$.

2) Expected total travel cost benefits: $\left.T T C B=p \bullet T T C S^{l}-(1-p) \bullet T T C L^{0}\right)$.

3) Expected total social benefits (TSB), the benefits to all road users from information. Therefore, the following equality holds by definition:

$$
T S B \equiv T D M B+T T C B
$$

4) Total "naïvely determined" willingness to pay for information (TNWTP), the area under the inverse demand function for information (up to $N_{E N}^{I n f}$ ), which is implied by (6).

5) Expected total "naïvely determined" external effects (TNEE), which can be expressed as

$$
T N E E=T S B-T N W T P=T T C B+T D M B-T N W T P
$$

The marginal measures are derived by taking derivative of 1)-5) above with respect to the number of informed drivers, $N_{E N}^{I n f}$, and will be denoted as $M D M B, M T C B, M S B$, $M N W T P$ and $M N E E$, respectively. ${ }^{15}$ When an additional driver gains access to information, $M S B$ comprises the total information benefits for this last driver, plus the changes in the travel cost and decision-making benefits, he brings to all the other drivers. As indicated by the relationship in (11), while $M T C B$ reflects what happens to the drivers only in terms of their travel costs, $M D M B$ captures the changes to all the already informed drivers in terms of their decision-making benefits, and, the decision-making benefits for the last driver himself. Alternatively, $M S B$ can be interpreted according to the relationship shown by the first equalities sign in (11). While MNWTP singles out the decision-making benefits that the last driver himself can obtain upon receiving information, what happens to all others is

\footnotetext{
${ }^{14}$ Note that all the benefit measures to be discussed are expected values over two states. However, the adjective 'expected' is dropped in the abbreviations for clarity.

${ }^{15}$ Actually $M W T P$ is a more appropriate term than $M N W T P$ for the marginal version of this measure, because it indeed correctly represents the marginal willingness to pay for information. However, we use MNWTP to maintain consistency in terminology. The same applies to MNEE.
} 
collected in $M N E E$, in terms of their total benefits (travel cost plus decision-making benefits).

The regime under road pricing pertains also to a comparison between equilibria with and without information, but with optimal fine congestion tolls imposed on the road in all cases. By doing so, marginal private costs will coincide with marginal social costs, and individually optimal behaviour is also in line with system optimality. In Appendix A.1.1, we provide calculations of the benefit measures and a mathematical representation of the model under the pricing regime.

In Figure 3, the measures described above are depicted as a function of information penetration. For the no road pricing scheme, we present two sets of results for different values $d$ and $a$, as indicated in the upper and middle panels in Figure 3 (corresponding to those we have chosen ourselves, and those from Emmerink (1998), respectively). The result associated with the less elastic parameters in Emmerink (1998) is interesting and helpful in deepening our understanding of the positive and negative external effects of information and congestion externalities, as will be discussed shortly. Under road pricing, only the outcome with the parameter set containing the 'our' $d$ and $a$ is produced, since both sets generate figures of similar patterns. Note that in all the figures, TNEE is not plotted in the left panels, neither are $M N E E$ and $M T C B$ in the right panels. This is because $T N E E$ is relatively of less importance than the other measures in our analysis, and the tendency in $M N E E$ and $M T C B$ can be easily inferred from other curves in the figures.

First of all, in the upper panels, the discrepancy between TSB (MSB) and TNWTP $(M N W T P)$ indicates the existence of information and congestion externalities. In the lower panels, $T S B^{*}\left(M S B^{*}\right)$ and $T N W T P^{*}\left(M N W T P^{*}\right)$ coincide, which can be naturally expected because of internalisation of the externalities.

The intersection of $M S B$ and $M N W T P$ in the middle right panel may be surprising at first sight, as one could expect that $M S B$ should always lie above MNWTP. An intersection is not a general result for the no road pricing regime, as is shown in the upper right panel where an intersection is absent. But the steeper slope of MSB than that of $M N W T P$ is a common result, caused by the same mechanism. To explain these, we need to examine three terms, $M D M B-M N W T P, M T C B$ and $M N E E$.

As can be seen in the right panels in Figure 3, there is a difference between $M N W T P\left({ }^{*}\right)$ and $M D M B(*)$. This difference reflects that more drivers getting equipped with ATIS will lead to losses for the already informed drivers in terms of their decision-making benefits. For an explanation, we refer to Figure 2.

In state 1 , for the marginally informed driver $N_{E N}^{1}$, his willingness to pay is equal to the decision-making benefits he enjoys, both equalling the difference (absolute value) between his willingness to pay for mobility and the actual travel costs on the road. However, when an additional driver gets informed, this additional driver will refrain from using the road due to the obvious losses otherwise, thus bringing the actual travel costs on the road down. Now the driver $N_{E N}^{1}$ becomes an intra-marginal informed driver and due to the decreased travel costs, his decision-making benefits will fall. Consequently, the driver $N_{E N}^{1}$ will be adversely affected in terms of his decision-making benefits. ${ }^{16}$ Similarly, in state 0 , an additionally informed driver will also create losses to the already informed drivers. Overall, there exist negative externalities with respect to drivers' decision-making benefits. This is proven in (A7) in the appendix.

\footnotetext{
${ }^{16}$ However, in terms of total benefits that this road user enjoys from informed road use, nothing changes (yet): he sticks to the policy of using the road in state 0 only, and this road user's surplus can still be written as $(1-p)\left(D\left(N_{E N}^{1}\right)-C^{0}\left(N_{E N}^{0}\right)\right.$.
} 
In Figure 3, TTCB is linear in the number of informed drivers, and MSB and $M D M B$ are parallel. This is consistent with the analytical result in (A9) in the appendix that $M T C B$ is a positive constant. This implies that each marginal informed driver creates identical travel cost benefits for the road system. In contrast with the negative externalities discussed above, this might be called a positive externality.

Graphically, MNEE is the difference between MSB and MNWTP, and reflects the changes in marginal external effects of information. Based on the discussion above, $M N E E$ is a combined outcome of positive externalities $(M T C B)$ and negative externalities $(M D M B-M N W T P)$. As more drivers join the information market, $M T C B$ stays invariant while $M D M B$ keeps falling, with the later arising from the fact that an extra informed driver affects more informed drivers at a higher penetration level than at a lower one. Particularly, in the middle right panel, MNEE by definition becomes negative after the intersection. It was found in the experiments that net effects of externalities depends on the relation between $a^{2}$ and $b^{0} b^{1}$. Other things being equal, when the former is smaller than the latter, $M S B$ and $M N W T P$ will have an intersection for MSB. Consequently, we can conclude that information and congestion externalities might generate positive or negative overall external effects for the road system, depending on the characteristics of the transport market. This result is due to the fact that the congestion externality is not internalised. Note that the optimal information price absent congestion pricing, implied by the value of $M N W T P$ at the point where $M S B$ intersects the horizontal axis, can be positive (in the middle panels) or negative (shown in the areas with the dotted lines in the upper panels). This price can be interpreted as a second-best congestion toll, which is nonzero only when congestion is not internalised through a direct toll (recall that the marginal costs of information, $\mu$, is set equal to zero. We will continue this issue in Section 3.2.

Next, we turn to examine question (i) raised in the beginning of this section. This entails an alternative decomposition of $M N E E$ into the marginal external effects for informed and uninformed drivers respectively.

Denoting the marginal external effects for uninformed drivers as $M N E E^{\text {unin }}$ and those for informed drivers as $M N E E^{\text {in }}, M N E E$ (by combing (A7) and (A9)) can now be written as

$$
M N E E=M N E E^{u n i n}+M N E E^{i n}
$$

where

$$
\begin{gathered}
M N E E^{\text {unin }}=-N_{E N}^{1}\left((1-p) \frac{\mathrm{d} C^{0}\left(N_{E N}^{0}\right)}{\mathrm{d} N_{E N}^{0}} \frac{\mathrm{d} N_{E N}^{0}}{\mathrm{~d} N_{E N}^{I n f}}\right. \\
\left.+p \frac{\mathrm{d} C^{1}\left(N_{E N}^{1}\right)}{\mathrm{d} N_{E N}^{1}} \frac{\mathrm{d} N_{E N}^{1}}{\mathrm{~d} N_{E N}^{I n f}}\right)>0 \\
M N E E^{i n}=-(1-p)\left(N_{E N}^{0}-N_{E N}^{1}\right) \frac{\mathrm{d} C^{0}\left(N_{E N}^{0}\right)}{\mathrm{d} N_{E N}^{0}} \frac{\mathrm{d} N_{E N}^{0}}{\mathrm{~d} N_{E N}^{I n f}}<0 .
\end{gathered}
$$


Figure 3 Impacts of information and congestion externalities on road users' information benefits $(\mu=F C=0)$
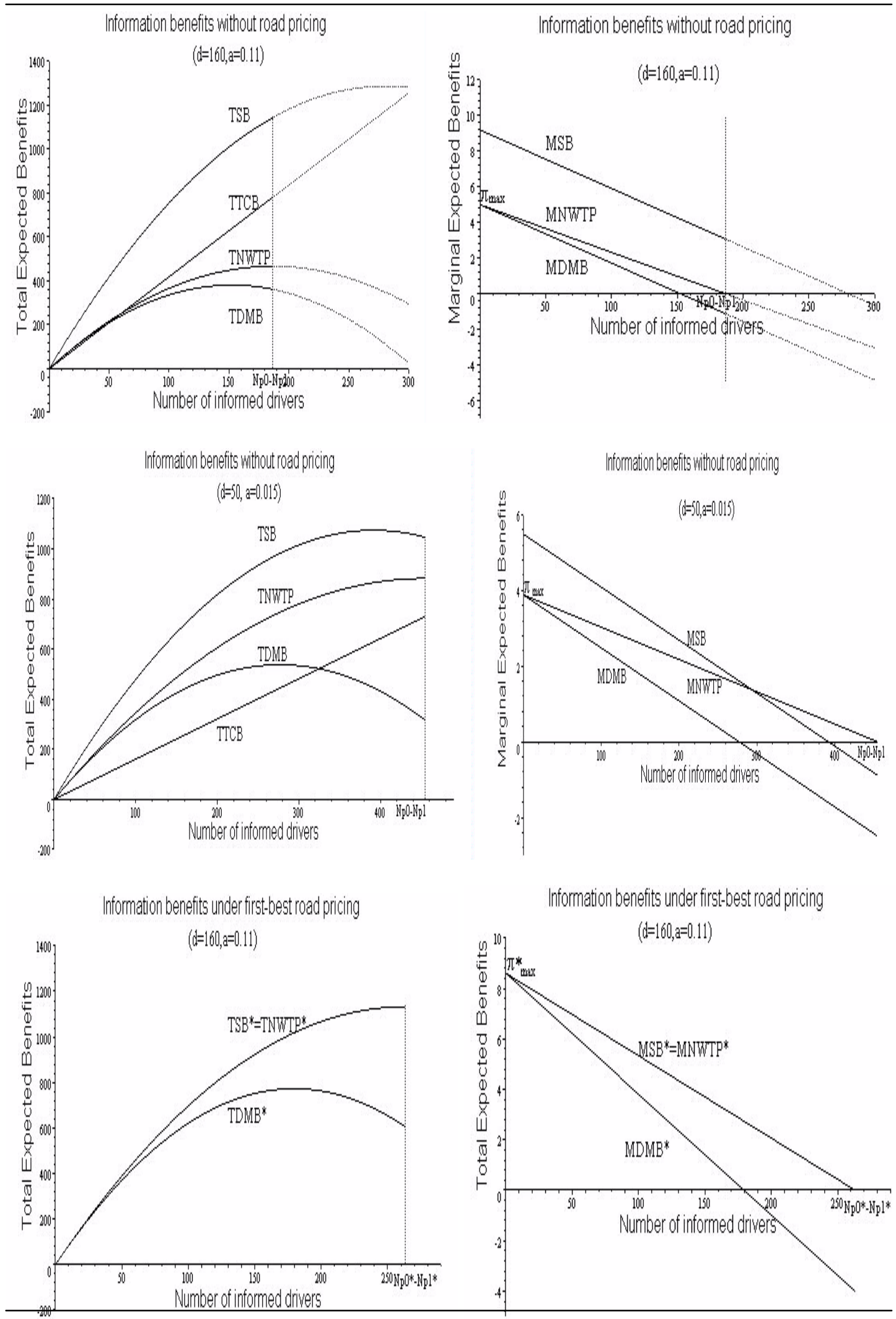

Note that $M N E E$ can be entirely captured by the changes in travel costs in each state. The fact that changes in decision-making benefits apparently can be written equally well in 
terms of changes in travel costs can be understood by a closer inspection of Figure 2. In state 1, $C_{E N}^{1}$ will fall as $N_{E N}^{I n f}$ increases. The implied fall in $T D M B^{l}$ for drivers between $N_{E N}^{1}$ and $N_{N}$ exactly cancels against the increase in $T T C S^{l}$ for these same drivers, with the result that the marginal external effects in state $1, M N E E^{l}$, can be written as the change in travel costs for $N_{E N}^{1}$ drivers. In state $0, C_{E N}^{0}$ will increase as $N_{E N}^{I n f}$ increases. The implied fall in $T D M B^{0}$ for drivers between $N_{N}$ and $N_{E N}^{0}$ will therefore equally well be measured as an increase in $T T C L^{0}$, with the result that the marginal external effects in state $0, M N E E^{0}$, can be written as the change in travel costs for $N_{E N}^{0}$ drivers.

$$
\frac{\mathrm{d} C^{0}\left(N_{E N}^{0}\right)}{\mathrm{d} N_{E N}^{0}} \frac{\mathrm{d} N_{E N}^{0}}{\mathrm{~d} N_{E N}^{\operatorname{Inf}}} \mathrm{in}(14) \text { reflects the marginal travel costs from an additionally }
$$

informed driver in state 0 . The multiplication by the number of uninformed drivers, $N_{E N}^{1}$, gives the marginal effects for all the uninformed drivers in that state. The second term in (14) reflects the marginal effects on travel costs for all the uninformed drivers in state 1 . In a similar fashion, the right-hand side of (15) captures the marginal effects on travel costs for all the informed drivers (between $N_{E N}^{1}$ and $N_{E N}^{0}$ ). Moreover, this expression shows that for the informed drivers, only the marginal travel costs in state 0 matter, because these drivers do not use the road in state 1 . As indicated by the signs of $M N E E^{\text {unin }}$ and $M N E E^{i n}$, $M N E E$ is still a sum of positive and negative externalities. In other words, there exist positive marginal external effects for uninformed drivers due to decreased expected travel costs. However, the informed drivers will suffer, as a result of increased travel costs in state 0 . Overall, increasing information penetration has adverse effects on informed drivers, not only in terms of their decision-making benefits, but also in terms of their total benefits (travel cost plus decision-making benefits). ${ }^{17}$ This result is in line with the findings from various modelling efforts by other researchers. That is, when a driver is already informed, having others informed does not have a positive effect (see Al-Deek and Kanafani, 1993; Levinson, 2003; Emmerink, 1998, Chapters 11 and 12).

\subsection{Monopoly information pricing and system efficiency}

According to the theory of monopoly pricing, a monopolist creates welfare losses for the commodity market it engages in by means of restricting output. In the present paper, since the markets for information and mobility are interrelated, the monopoly pricing can be expected to have a direct impact on road use. Therefore, our objective in this section is to study system efficiency in response to the monopoly price formed in the information market. Here, we choose to examine system welfare relative to the no information case, or, welfare gain from information, which we denote by $W G .{ }^{18}$ In addition, it is useful to consider an information pricing scheme that maximizes $W G$. That is, we assume a regulator that charges a price $\pi$ for information so that optimal system performance can be

\footnotetext{
${ }^{17}$ See the discussion above Figure 2 and note the complication that the drivers between $N_{E N}^{1}$ and $N_{N}$ enjoy not only decision-making benefits, but also travel cost benefits.

${ }^{18}$ By doing this, we can make a direct comparison between Figures 3 and 4, which is helpful in understanding the impacts of the pricing schemes. System welfare differs from $W G$ by the amount of the welfare in the no information case, which equals

$p\left(\int_{0}^{N_{N}} D(n) d n-C^{1}\left(N_{N}\right) N_{N}\right)+(1-p)\left(\int_{0}^{N_{N}} D(n) d n-C^{0}\left(N_{N}\right) N_{N}\right)$, a constant. Therefore, it makes no difference whether to use system welfare or $W G$ in calculating $\pi_{o p}$ and $\pi_{o p}^{*}$.
} 
achieved. We name this a public information pricing regime, with the corresponding price denoted by $\pi_{o p}$. The expression for $\pi_{m}$ is given in (10) in Section 2.2, and $\pi_{o p}$ in (A17).

Similar to Section 3.1, we also consider the two sets of parameters that differ in the values of $d$ and $a$, as shown in the upper and lower panels in Figure 4 respectively. Further, the case of first-best congestion pricing is also examined, which enables us to analyse the welfare implications of monopoly pricing when congestion externalities are internalised.

Figure 4 Welfare gain and monopoly information pricing $(\mu=F C=0)$
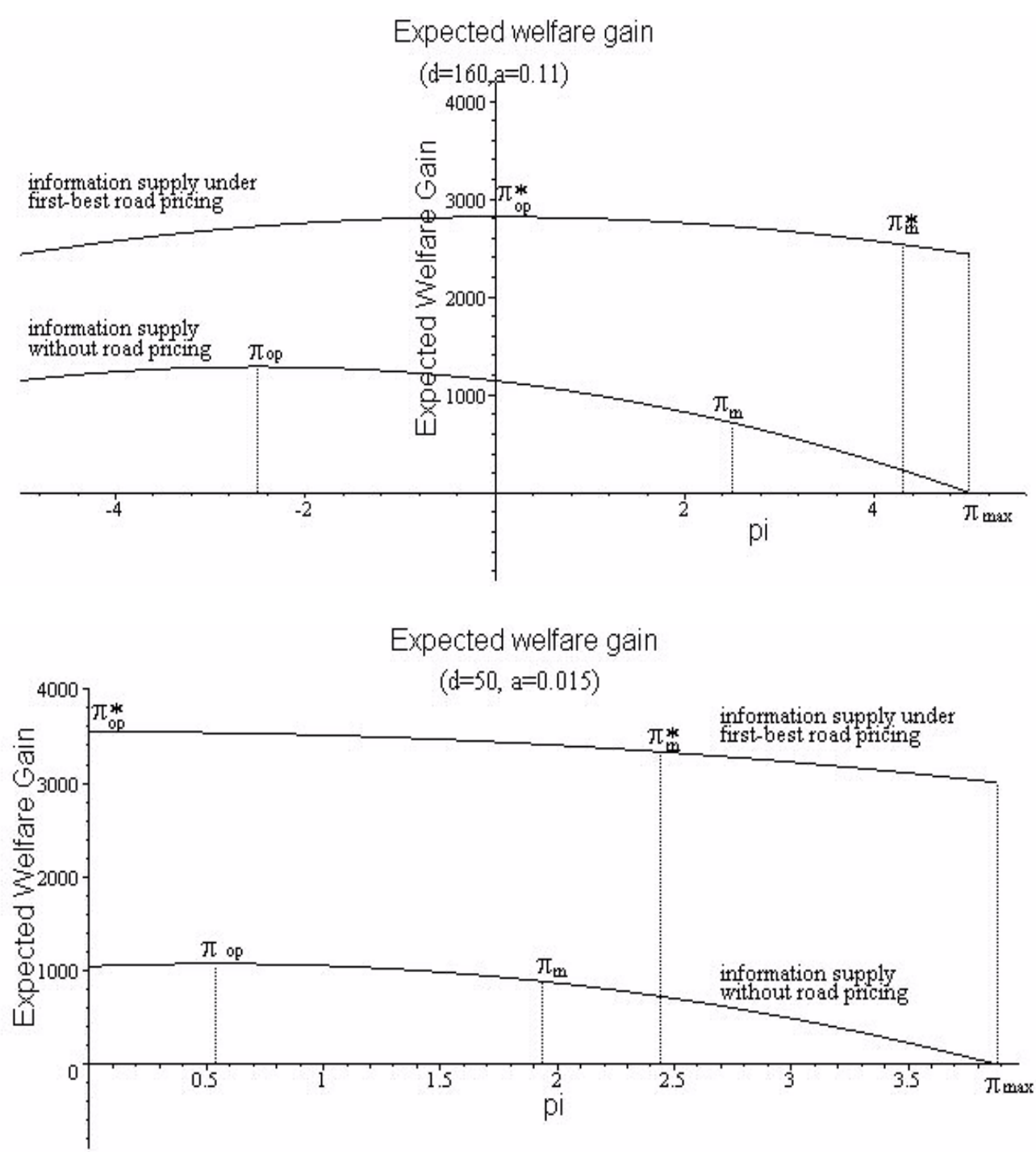

The two panels in Figure 4 show $W G$ as a function of the information price, $\pi$. In each panel, the upper curve reflects the case with road pricing, and the lower one without. $\mu$ and $F C$ are set to zero in the figure for ease of interpretation. The lower curves are in fact transformations of the TSB curves in Figure 3, but with $W G$ now expressed as a function of $\pi$, instead of market penetration. In these curves, each $\pi_{m}$ corresponds to a lower level of $W G$ relative to $\pi_{o p}$. In Appendix A.2.1, it is proven that under the rather weak assumption of free flow travel costs being equal in both states $\left(k^{0}=k^{l}\right), \pi_{m}$ always exceeds $\pi_{o p}$ within the range $\left[0, \pi_{\max }\right]$. In the upper curves, $\pi_{m}^{*}$ also exceeds $\pi_{o p}^{*}$, with the result that welfare is lower. Therefore, monopoly pricing is a less attractive option with respect to system efficiency in both cases, which confirms intuition. 
It is also useful to pay some attention to the result that $W G$ is maximized at a nonzero price in the no road pricing case (a negative price, which suggests a subsidy that will be discussed in next section) ${ }^{19}$ In contrast, $W G^{*}$ is maximized at zero ( $\mu$ in the general case) with road pricing implemented: as the distortions on the road are corrected, the optimal information price should be set equal to the marginal costs, or, the competitive price (see Appendix A.2.2). This reflects that information pricing (or subsidies) might act as a second-best charge in handling congestion externalities. However, as pointed out in Verhoef, Emmerink, Nijkamp and Rietveld (1996), information does not exhibit the potential of achieving maximal social welfare (under fine congestion pricing). This is confirmed by the vertical difference between the two curves in each panel. The remark also explains why the second-best effects of information pricing have disappeared in the upper curves: the more effective first-best congestion pricing has taken over the task of dealing with congestion and completely internalised the externalities.

\subsection{Subsidising information supply}

Section 3.2 introduced the potential of information subsidy in alleviating congestion. This section discusses this issue in greater detail.

Generally speaking, three archetype kinds of subsidy can be envisaged as policy instruments in regulating our information market, namely, a user subsidy, a producer subsidy per unit of output, and a lump-sum subsidy to the producer. Because a lump-sum subsidy has no immediate influence on a firm's marginal production decisions given its existence, we concentrate on a user and a producer subsidy, which do bear a direct relationship with system efficiency. In particular, we wish to find out whether there are differences between a user and a producer subsidy regarding their impacts on welfare gain from information.

These subsidies affect system efficiency because they enter the information buyers' and the monopolist's decision-making processes. In the following, we give the mathematical representation of the agents' maximizing behaviour when being subsidized. Given the price of information, expression (16) constitutes the equilibrium condition in each state of the road when the users are subsidized:

$$
\begin{aligned}
& (1-p)\left(D\left(N_{E N}^{0}\right)-C^{0}\left(N_{E N}^{0}\right)\right)=\pi-u s \\
& p\left(C^{l}\left(N_{E N}^{1}\right)-D\left(N_{E N}^{1}\right)\right)=\pi-u s
\end{aligned}
$$

where $u s$ refers to user subsidy. That is, $u s$ subtracts a positive quantity from the price of the product so that the information systems now become cheaper than $\pi$. The profitmaximizing monopolist would take a producer subsidy into account in the following way,

$$
\operatorname{Max}_{\pi} \pi \bullet N_{E N}^{\operatorname{Inf}}(\pi)-\left(F C+(\mu-p s) \bullet N_{E N}^{I n f}(\pi)\right)
$$

where $p s$ stands for producer subsidy. (17) states that upon receiving the subsidy, the monopolist now faces a lower cost than the original one for each unit of ATIS produced. It turns out that the consumer and producer subsidy have, as expected, identical results (see Appendix A.3).

\footnotetext{
${ }^{19}$ It is found in the experiments that the sign of $\pi_{o p}$ depends on the comparison between $a$ and $b^{0} b^{l}$ and on the size of $\mu$. The greater the $a$, the more likely that $\pi_{o p}$ will be negative. $\mu$ is also negatively related with $\pi_{\mathrm{op}}$.
} 

pricing, $\mu=F C=0, d=160$ and $a=0.11$ )

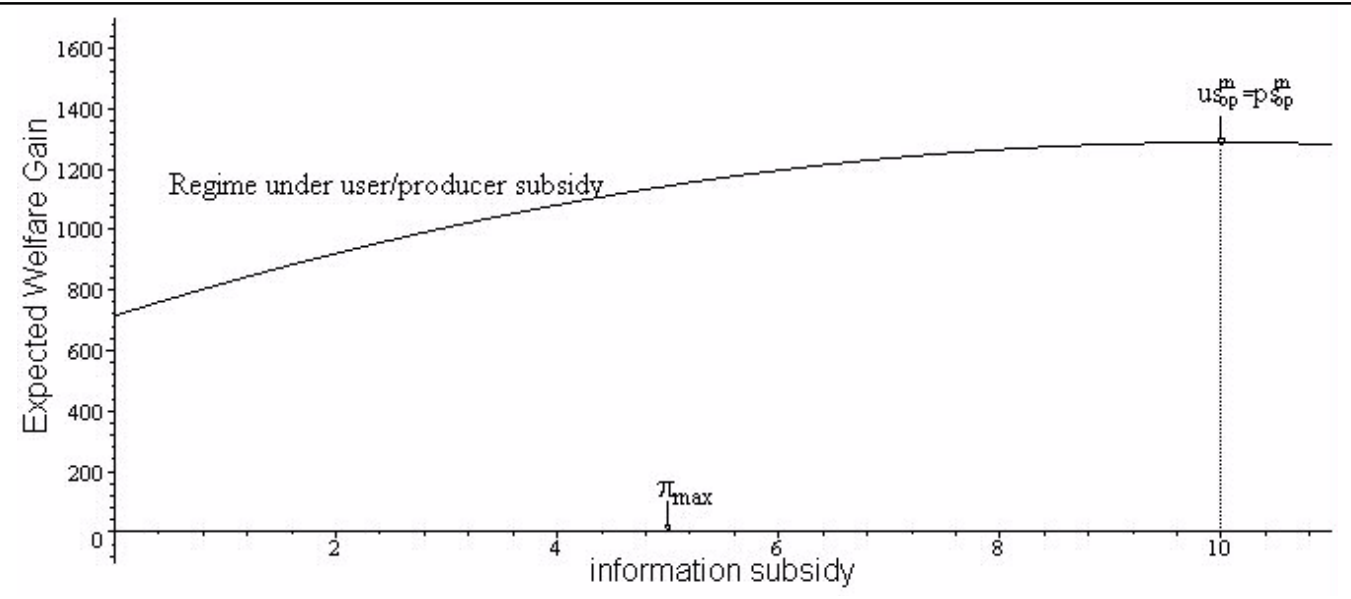

Experiment results are shown in Figure 5, where $\mu$ and $F C$ are again assumed equal to zero. In the figure, one single curve depicts $W G$ as a function of the user/producer subsidy, given the monopolist' pricing strategy. This reflects that $p s$ and $u s$ are fully equivalent in their impacts. By comparing Figures 4 and 5, it is also easily seen that subsidizing information can lead to the same level of optimal $W G$ as information pricing does. Their optimal values, $u s_{o p}^{m}$ and $p s_{o p}^{m}$, are therefore also equal.

\subsection{Some factors influencing relative system efficiency under the monopolist' pricing strategy}

In this section, we vary the value of some parameters in the model, to gain some insight into the impacts of these parameters on the relative efficiency of monopolistic information supply. The parameters of interest are: the probability of low road capacity $p$, demand elasticity for mobility, and marginal information cost $\mu$. We conduct this analysis by using an efficiency index $\omega$ (see Arnott, et al., 1991; Emmerink 1998). It indicates the welfare gain from information on a non-optimally priced road network as a proportion of the (theoretically) highest achievable welfare gain.

The index $\omega$ is then defined as:

$$
\omega=\frac{\text { Welfare Gain (policy considered }}{\text { Welfare Gain (system optimum) }}
$$

where 'policy considered' can refer to public information pricing and private information pricing ( $\pi_{o p}$ and $\pi_{m}$ in Figure 4$)$. According to the definition, $\omega$ cannot exceed 1.

Experiment results with respect to the impacts of the parameters on relative system efficiency are plotted in the lower panels in Figures 6 and 7 respectively. For comparison purposes, the case of 'public pricing' as discussed in Section 3.2 is included in the lower panels as well. 
Figure 6 Impacts of $p$ and $\mu$ on the monopolist's profit, and on $\omega$ for public and private information pricing (base case: $\mu=0.5, F C=100, d=160$ and $a=0.11$ )
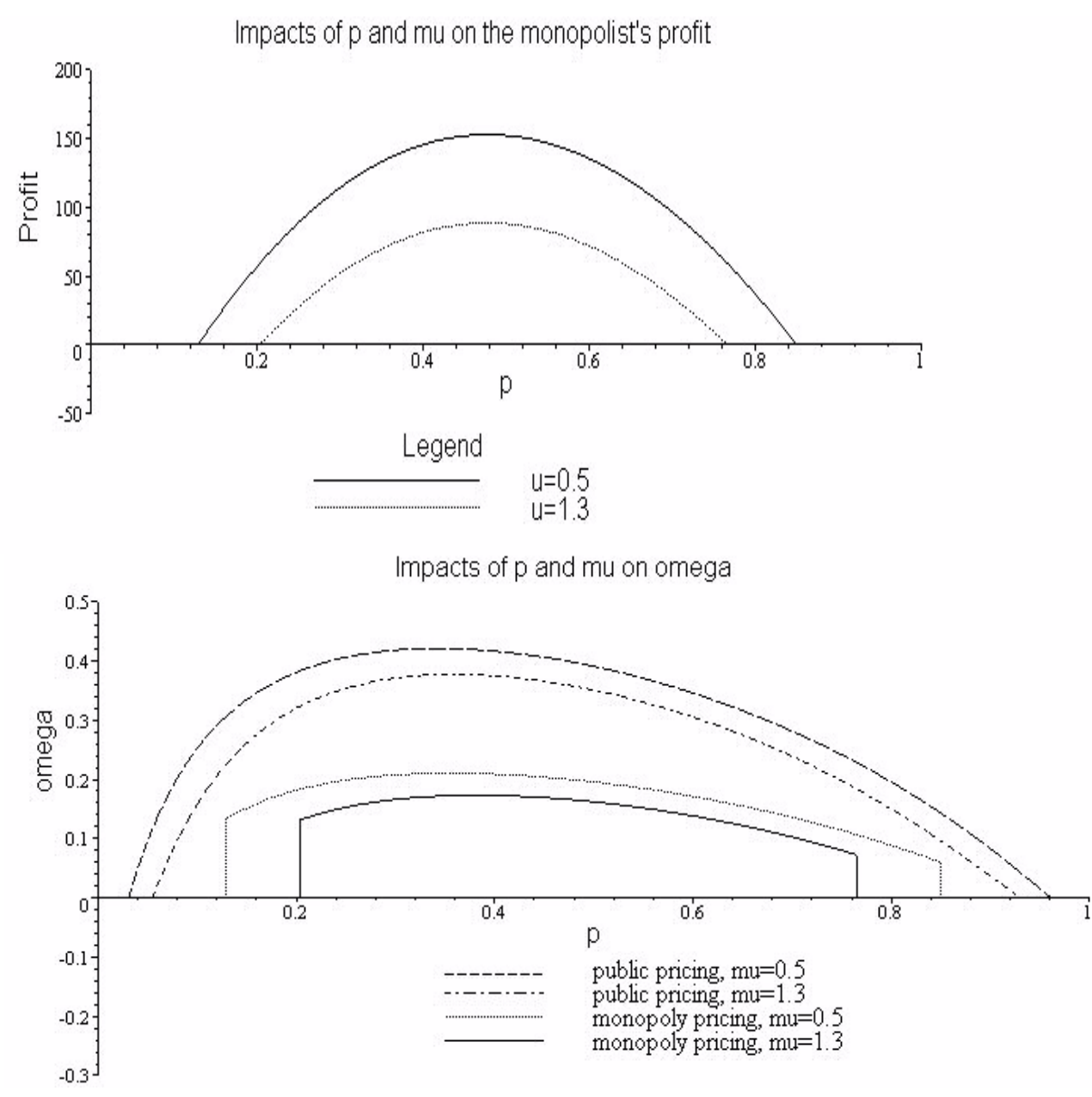

Figure 6 combines the impacts of $p$ and $\mu$ on the monopolist's profit and on $\omega$. First, in the upper panel, information is more profitable for the monopolist at relatively high levels of uncertainty (so, intermediate levels of $p$ ); that is, the users are more willing to pay for the information, the more unsure they are about the road conditions. This result appeals to intuition. A similar pattern can be found in the lower panel: information is more valuable for the road system as a whole when uncertainty on the road is relatively large. Second, the comparison between the two pricing regimes at each level of $\mu$ shows that the monopolist' pricing strategy is not as attractive an option as the public pricing case on efficiency grounds, irrespective of the levels of uncertainty ${ }^{20}$. Third, and expectedly, the higher the costs of information, the lower is the profit for the monopolist, as is clear in the upper panel. In the lower panel, $\mu$ affects $\omega$ in the same manner: a lower $\mu$ results in a larger $\omega$ in both pricing schemes. Finally, it is observed in the upper panel that the range of $p$ over which profit exceeds zero shrinks when $\mu$ increases, which is due to the existence of fixed and variable costs of information provision. As a result, we only consider the values

\footnotetext{
${ }^{20}$ To ensure the correctness of the experiment results, crossing-checking of experiments related to Figure 4 and Figure 6 is carried out. That is, the ratio $\pi_{m} / \pi_{o p}$ is calculated for three combinations of $\mu$ and $F C$, namely, 1) $\mu=F C=0,2) \mu=0.5, F C=100$, and 3$) \mu=1.3, F C=100$. For each combination, the ratios calculated from each experiment are exactly the same, being $0.55,0.49$ and 0.43 respectively.
} 
of $p$ that correspond to a positive profit when drawing the graph of $\omega$ in the monopoly scheme. The explanation of the negative profit necessitates a view on the joint impacts of $\mu$ and $p$ on profit. Suppose $p$ is very small (in the neighbourhood of 0 ). Then, a very low $\pi_{\max }$ will follow (see (A21) and note that $\pi_{\max }^{0}=\pi_{\max }^{1}=\pi_{\max }$ ) and it will fall below any positive value of $\mu$ for sufficiently small $p$. In these circumstances, the monopolist cannot make profits. $^{21}$

The impacts of demand elasticity for mobility on the monopolist's profit and on $\omega$ in both pricing schemes are illustrated in Figure 7. To construct this figure, the parameters $a$ and $d$ are altered in such a way that the curve of inverse demand for mobility tilts around the equilibrium of Model $\mathrm{N}$ (point $\left(N_{N}, C_{N}\right)$ ). This is used to represent changes in demand elasticilites for mobility. ${ }^{22}$ The elasticities vary from high to low along the horizontal axis (the slope of $D(N)$ (absolute value) grows from left to right). ${ }^{23}$

First of all, in the upper panel of Figure 7, the monopolist's profit from information decreases when demand for mobility changes from elastic to inelastic. Intuitively, this might be due to the fact that inelastic demand for mobility reflects drivers' heavy reliance on the road for their trips, which in turn suggests limited value of information for the drivers (not many drivers would avoid state 1 when being informed, nor would many drivers be attracted to only driving in state 0 when being informed). Therefore, the more elastic the demand for road use, the higher will the monopolist's profits be. Profit from information will turn into negative when the elasticities are sufficiently low. This result appears to be in contrast with standard results where monopolists benefit from inelastic rather than elastic demand. The difference arises because the demand for information is derived from the demand for road use. In Appendix A.4, we give detailed explanation for this.

Next, we shift to the $\omega$ 's, which are also restricted to the interval of positive profit. In the lower panel, the $\omega$ 's from the two pricing schemes converge in the extreme case where demand for mobility approaches perfect elasticity. This stems from the converging prices in the two schemes (see (10) and (A17)). As the slope of $D(N)$ (absolute value) grows along the horizontal axis, the $\omega$ 's begin to diverge. As shown, relative system efficiency first increases and then decreases: there exists a level of elasticity that maximizes $\omega$. Moreover, at any nonzero elasticities, monopoly information pricing remains relatively inefficient compared to the public pricing case.

\footnotetext{
${ }^{21}$ This phenomenon can be found in the public pricing regime as well. This is related with the fact that information costs enter the calculation of system welfare. The reasoning is the same as in the negative profit case.

${ }^{22}$ The equilibrium Model $\mathrm{N}$ is used as a reference point and the elasticities referred to are the corresponding point elasticities. Therefore, changes in the slope of demand for mobility are representative of changes in the elasticities if the demand for mobility tilts around this intersection. The reason to do so is to avoid very small (large) levels of road usage when demand approaches complete (in)elasticity.

${ }^{23}$ In the lower panel, it is noticed that because the position of the intersection point changes with $p$ (see (1) in Section 2.2), the equilibrium elasticities of the demand curves differ slightly in $p$ even with the same slope of $D(N)$. Therefore, only the curves with the same probability are strictly comparable. For example, the reference point $\left(N_{N}, C_{N}\right)$ is $(1067,42.67)$ for $p=0.25$, and $(1037,45.93)$ for $p=0.4$. The corresponding elasticities at slope 0.11 are -0.36 and -0.40 respectively (absolute value).
} 
Figure 7 Impacts of demand elasticity for mobility on the monopolist's profit and $\omega$ (base case: $\mu=0.5, F C=100, d=160$ and $a=0.11$ )
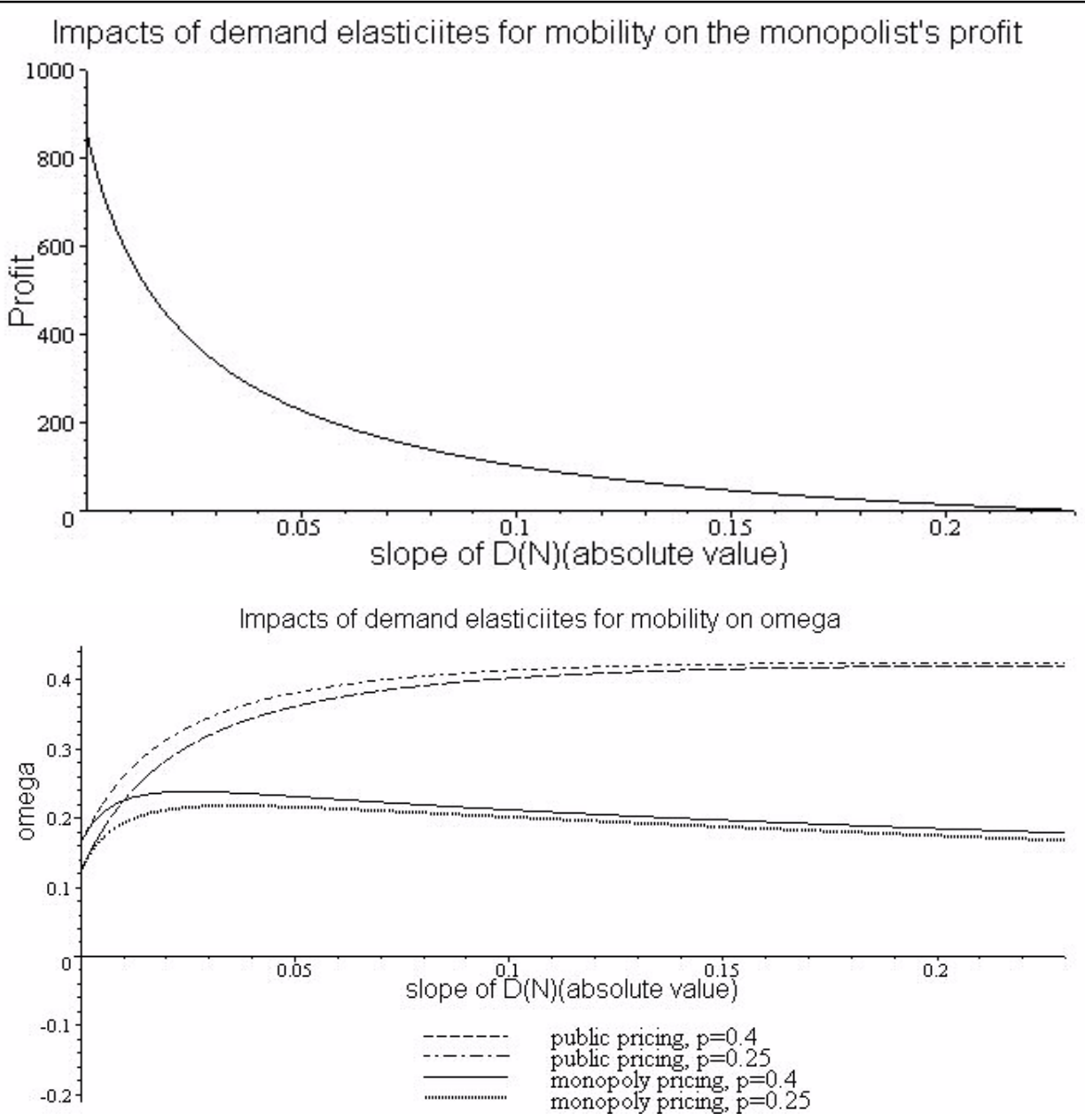

\section{Conclusions}

We have constructed a model system that examines a transport market and a monopolist market for ATIS. Using this model, we investigated the welfare properties of the information market. In particular, we assessed the impacts of monopoly information pricing on system efficiency.

Our results show that due to the existence of positive and negative external effects of information and congestion externalities, increasing market penetration of information will adversely affect the already informed drivers. In contrast, the uninformed drivers will be better irrespective of the levels of market penetration.

Further, the monopoly information pricing corresponds to a lower level of system efficiency, compared to a pricing scheme where welfare gain from information is maximized. When first-best fine congestion pricing is imposed on the road, the distortion from the market power persists. This is because in both pricing schemes, the monopolist charges a too high price to pursue profit maximization.

Third, we have found that under monopolist's pricing strategy, a subsidy on the information market can be adopted by the government to reach a second-best system optimum of road use.

Finally, some parameters in the model system have turned out important for the monopolist profit as well as for relative system efficiency. For instance, a high level of uncertainty in the system makes information more valuable for both the road users and the 
monopolist. Marginal costs of information have been found to be negatively associated with the monopolist's profit and relative system efficiency. A seemingly surprising outcome involving demand elasticities for mobility is that information is more profitable for the monopolist at relatively inelastic demand. This is due to the derived characteristics of the demand function for information and can be intuitively understood as arising from drivers' unresponsiveness to changes in travel costs. However, for the system as whole, the highest relative efficiency gain is achieved at an intermediate elasticity. 


\section{References}

Al-Deek, H. and A. Kanafani (1993). Modelling the benefits of advanced traveller information systems in corridors with incidents. Transportation Research part C, Vol.1, No. 4, pp. 303-324.

Arnott, R., A.de Palma and R. Lindsey (1991). Does providing information to drivers reduce traffic congestion? Transportation Research, 25A, 309-318.

Emmerink, R. H. M. (1998). Information and Pricing Road in Transport. Berlin, Springer.

Emmerink, R. H. M., E. T. Verhoef, P. Nijkamp and P. Rietveld (1996).

Endogenising demand for information in road transport. Annals of Regional Science (30), 201-222.

Levinson, D. 2003. The value of advanced traveler information systems for route choice. Transportation Research part C, 11(1): 75-87.

Noonan, J. and O. Shearer (1998). Intelligent Transportation Systems Field Operational Test Cross-Cutting Study Advanced Traveler Information Systems. Report prepared for U.S. Department of Transportation, Federal Highway Administration, Washington, D.C.

Verhoef, E.T., R. H. M. Emmerink, P.Nijkamp and P. Rietveld (1995). Second-best regulation of road transport externalities, Journal of Transport Economics and Policy, 29,147- 167. 


\section{Appendices}

\section{A.1 Information and congestion externalites}

In this appendix, we first give the calculations of the benefit measures as described in Section 3.1. The proof of positive/negative information and congestion externalities is presented as well. Second, we derive the demand function for information in the regulatory scheme of road pricing. The benefits from information in this regime can be computed in a similar way, which we do not provide.

\section{A.1.1 Calculations of information benefits without tolling}

Assuming the numbers of road users are $N_{E N}^{0}$ and $N_{E N}^{1}$ in state 0 and 1 respectively, $T D M B, T T C B$ and $T N W T P$ are

$$
\begin{aligned}
& T D M B=(1-p)\left(\int_{N_{N}}^{N_{E N}^{0}} D(n) d n-C^{0}\left(N_{E N}^{0}\right)\left(N_{E N}^{0}-N_{N}\right)\right) \\
& +p\left(C^{1}\left(N_{E N}^{1}\right)\left(N_{N}-N_{E N}^{1}\right)-\int_{N_{E N}^{I}}^{N_{N}} D(\mathrm{n}) d n\right) \\
& T T C B=\left(p\left(C^{1}\left(N_{N}\right)-C^{1}\left(N_{E N}^{1}\right)-(1-p)\left(C^{0}\left(N_{E N}^{0}\right)-C^{0}\left(N_{N}\right)\right)\right) N_{N} .\right. \\
& T N W T P=(1-p)\left(\int_{N_{N}}^{N_{E N}^{0}} D(n) d n-\int_{N_{N}}^{N_{E N}^{0}} C^{0}(n) d n\right)+p\left(\int_{N_{E N}^{1}}^{N_{N}} C^{1}(n) d n-\int_{N_{E N}^{1}}^{N_{N}} D(n) d n\right) .
\end{aligned}
$$

$T S B$ and TNEE can be calculated according to (11) and (12).

To derive the marginal measures, the total terms need be written as a function of $N_{E N}^{I n f}$. However, because information price $\pi$ is the only variable channelling the two states, we use the (explicit) formulae (5.a) and (5.b) and the inverse demand function for information to achieve this. The marginal measures are then obtained by taking derivative of the total measures with respect to $N_{E N}^{I n f}$. These are given below:

$$
\begin{aligned}
M D M B= & (1-p)\left(D\left(N_{E N}^{0}\right) \frac{\mathrm{d} N_{E N}^{0}}{\mathrm{~d} N_{E N}^{I n f}}-\left(N_{E N}^{0}-N_{N}\right) \frac{\mathrm{d} C^{0}\left(N_{E N}^{0}\right)}{\mathrm{d} N_{E N}^{0}} \frac{\mathrm{d} N_{E N}^{0}}{\mathrm{~d} N_{E N}^{I n f}}\right. \\
& \left.-C^{0}\left(N_{E N}^{0}\right) \frac{\mathrm{d} N_{E N}^{0}}{\mathrm{~d} N_{E N}^{I n f}}\right)+p\left(\left(N_{N}-N_{E N}^{1}\right) \frac{\mathrm{d} C^{1}\left(N_{E N}^{1}\right)}{\mathrm{d} N_{E N}^{1}} \frac{\mathrm{d} N_{E N}^{1}}{\mathrm{~d} N_{E N}^{I n f}}\right. \\
& \left.-C^{1}\left(N_{E N}^{1}\right) \frac{\mathrm{d} N_{E N}^{1}}{\mathrm{~d} N_{E N}^{I n f}}+D\left(N_{E N}^{1}\right) \frac{\mathrm{d} N_{E N}^{1}}{\mathrm{~d} N_{E N}^{I n f}}\right) . \\
M T C B= & (1-p)\left(D\left(N_{E N}^{0}\right) \frac{\mathrm{d} N_{E N}^{0}}{\mathrm{~d} N_{E N}^{I n f}}-N_{E N}^{0} \frac{\mathrm{d} C^{0}\left(N_{E N}^{0}\right)}{\mathrm{d} N_{E N}^{0}} \frac{\mathrm{d} N_{E N}^{0}}{\mathrm{~d} N_{E N}^{I n f}}\right. \\
- & \left.C^{0}\left(N_{E N}^{0}\right) \frac{\mathrm{d} N_{E N}^{0}}{\mathrm{~d} N_{E N}^{I n f}}\right)+p\left(D\left(N_{E N}^{1}\right) \frac{\mathrm{d} N_{E N}^{1}}{\mathrm{~d} N_{E N}^{I n f}}\right. \\
- & \left.N_{E N}^{1} \frac{\mathrm{d} C^{1}\left(N_{E N}^{1}\right)}{\mathrm{d} N_{E N}^{1}} \frac{\mathrm{d} N_{E N}^{1}}{\mathrm{~d} N_{E N}^{I n f}}-C^{1}\left(N_{E N}^{1}\right) \frac{\mathrm{d} N_{E N}^{1}}{\mathrm{~d} N_{E N}^{I n f}}\right) .
\end{aligned}
$$




$$
\begin{aligned}
M N W T P & =(1-p)\left(D\left(N_{E N}^{0}\right) \frac{\mathrm{d} N_{E N}^{0}}{\mathrm{~d} N_{E N}^{I n f}}-C^{0}\left(N_{E N}^{0}\right) \frac{\mathrm{d} N_{E N}^{0}}{\mathrm{~d} N_{E N}^{I n f}}\right) \\
& +p\left(-C^{1}\left(N_{E N}^{1}\right) \frac{\mathrm{d} N_{E N}^{1}}{\mathrm{~d} N_{E N}^{I n f}}+D\left(N_{E N}^{1}\right) \frac{\mathrm{d} N_{E N}^{1}}{\mathrm{~d} N_{E N}^{I n f}}\right) .
\end{aligned}
$$

The existence of negative and constant positive effects of information and congestion externalities is proven below.

$$
\begin{aligned}
M D M B-M N W T P & =-(1-p)\left(N_{E N}^{0}-N_{N}\right) \frac{\mathrm{d} C^{0}\left(N_{E N}^{0}\right)}{\mathrm{d} N_{E N}^{0}} \frac{\mathrm{d} N_{E N}^{0}}{\mathrm{~d} N_{E N}^{I n f}} \\
& +p\left(\left(N_{N}-N_{E N}^{1}\right) \frac{\mathrm{d} C^{1}\left(N_{E N}^{1}\right)}{\mathrm{d} N_{E N}^{1}} \frac{\mathrm{d} N_{E N}^{1}}{\mathrm{~d} N_{E N}^{I n f}} .\right.
\end{aligned}
$$

Using the assumption that $\mathrm{d} C^{l}(N) / \mathrm{d} N \geq \mathrm{d} C^{0}(N) / \mathrm{d} N>0$ for all $N$, and the result that $\frac{\mathrm{d} N_{E N}^{0}}{\mathrm{~d} N_{E N}^{I n f}}=\frac{\mathrm{d} N_{E N}^{0}}{\mathrm{~d} \pi} \frac{\mathrm{d} \pi}{\mathrm{d} N_{E N}^{I n f}}>0$ but $\frac{\mathrm{d} N_{E N}^{1}}{\mathrm{~d} N_{E N}^{I n f}}=\frac{\mathrm{d} N_{E N}^{1}}{\mathrm{~d} \pi} \frac{\mathrm{d} \pi}{\mathrm{d} N_{E N}^{I n f}}<0$, it is obvious that (A7) is smaller than zero - negative externalities.

$$
\begin{aligned}
M T C B= & -N_{N}\left((1-p) \frac{\mathrm{d} C^{0}\left(N_{E N}^{0}\right)}{\mathrm{d} N_{E N}^{0}} \frac{\mathrm{d} N_{E N}^{0}}{\mathrm{~d} N_{E N}^{I n f}}\right. \\
& \left.+p \frac{\mathrm{d} C^{1}\left(N_{E N}^{1}\right)}{\mathrm{d} N_{E N}^{1}} \frac{\mathrm{d} N_{E N}^{1}}{\mathrm{~d} N_{E N}^{I n f}}\right) .
\end{aligned}
$$

Applying the explicit functions for $C^{0}, C^{1}, N_{E N}^{0}(\pi)$ and $N_{E N}^{1}(\pi)$, and the assumptions that $a>0, b^{l}>b^{0}>0$ and $\frac{\mathrm{d} \pi}{\mathrm{d} N_{E N}^{I n f}}<0$,

$$
\begin{aligned}
M T C B & =-N_{N} \frac{a\left(b^{1}-b^{0}\right)}{\left(a+b^{0}\right)\left(a+b^{1}\right)} \frac{\mathrm{d} \pi}{\mathrm{d} N_{E N}^{\operatorname{Inf}}} \\
& =\mathrm{a} \text { positive constant. }
\end{aligned}
$$

That is, constant positive externalities.

\section{A.1.2 Demand for information under first-best road pricing}

In this regime, the equilibrium in Model EN comprise of the following two equalities:

$$
\begin{aligned}
& (1-p)\left(D\left(N_{e n}^{0^{*}}\right)-C^{0}\left(N_{e n}^{0^{*}}\right)-f^{0}\right)=\pi \\
& p\left(C^{1}\left(N_{e n}^{1^{*}}\right)+f^{1}-D\left(N_{e n}^{1^{*}}\right)\right)=\pi
\end{aligned}
$$

where $\mathrm{f}^{0}$ and $\mathrm{f}^{1}$ represent optimal congestion tolls in each state (fine tolling); that is, 


$$
\begin{aligned}
& f^{0}=\frac{\mathrm{d} C^{0}\left(N_{E N}^{0^{*}}\right)}{\mathrm{d} N_{E N}^{0^{*}}} N_{E N}^{0^{*}} \\
& f^{1}=\frac{\mathrm{d} C^{1}\left(N_{E N}^{1^{*}}\right)}{\mathrm{d} N_{E N}^{1^{*}}} N_{E N}^{1^{*}} .
\end{aligned}
$$

Here, the optimal fine fees are equal to marginal external costs at $N_{E N}^{0^{*}}$ and $N_{E N}^{1^{*}}$, the traditional expression that reflects the external costs of congestion in each state.

Given $\pi$, the equilibrium road usage in each state is

$$
\begin{aligned}
& N_{\mathrm{EN}}^{0^{*}}(\pi)=\frac{d-k^{0}}{a+2 b^{0}}-\frac{\pi}{(1-p)\left(a+2 b^{0}\right)} \\
& N_{\mathrm{EN}}^{1^{*}}(\pi)=\frac{d-k^{l}}{a+2 b^{l}}+\frac{\pi}{p\left(a+2 b^{l}\right)}
\end{aligned}
$$

The demand for information $N_{E N}^{I n f^{*}}$ can also derived, which is

$$
\begin{aligned}
& N_{E N}^{I^{*}}(\pi)=N_{E N}^{0^{*}}(\pi)-N_{E N}^{1^{*}}(\pi) \\
& \quad=\frac{d-k^{0}}{a+2 b^{0}}-\frac{d-k^{1}}{a+2 b^{l}}-\pi\left(\frac{a+2\left((1-p) b^{0}+p b^{1}\right)}{p(1-p)\left(a+2 b^{0}\right)\left(a+2 b^{1}\right)}\right) .
\end{aligned}
$$

$T D M B^{*}, T T C B^{*}, T N W T P^{*}$ and the corresponding marginal terms can be easily computed in a similar way as in the no tolling case.

\section{A.2 Welfare -maximizing information price: with and without road pricing}

In this appendix, we derive the information price that maximizes social welfare gain from information. Both the regimes with and without road pricing are considered. In the no pricing regime, we also conduct a comparison between $\pi_{m}$ and $\pi_{o p}$ and will show that $\pi_{m}>$ $\pi_{o p}$ for $\mu \in\left[0, \pi_{\max }\right]$.

\section{A.2.1 No road pricing case}

First, we derive $\pi_{o p}$. The welfare gain in the road system due to information is written as the $T S B$, as discussed above, minus information costs; that is,

$$
W G=T S B-\mu \bullet\left(N_{E N}^{0}-N_{E N}^{1}\right)-F C
$$

Then the maximization problem can be stated as following:

$$
\max _{\pi} W G
$$

subject to the constraints in (4).

The maximization problem is solved by first substituting (5.a) and (5.b) into (A14) to express $W G$ as a function of $\pi$. Then $\pi_{o p}$ is computed from the following first-order condition: 


$$
\begin{aligned}
\frac{\mathrm{d} W G}{\mathrm{~d} \pi}= & (1-p) \bullet\left(D\left(N_{E N}^{0}\right)-C^{0}\left(N_{E N}^{0}\right)\right) \frac{\mathrm{d} N_{E N}^{0}\left(\pi_{o p}\right)}{\mathrm{d} \pi} \\
& -\mathrm{p} \bullet\left(C^{1}\left(N_{E N}^{1}\right)-D\left(N_{E N}^{1}\right)\right) \frac{\mathrm{d} N_{E N}^{1}\left(\pi_{o p}\right)}{\mathrm{d} \pi} \\
& -(1-p) \frac{\mathrm{d} C^{0}\left(N_{E N}^{0}\right)}{\mathrm{d} N_{E N}^{0}} \frac{\mathrm{d} N_{E N}^{0}\left(\pi_{o p}\right)}{\mathrm{d} \pi} N_{E N}^{0}\left(\pi_{o p}\right) \\
& -p \frac{\mathrm{d} C^{1}\left(N_{E N}^{1}\right)}{\mathrm{d} N_{E N}^{1}} \frac{\mathrm{d} N_{E N}^{1}\left(\pi_{o p}\right)}{\mathrm{d} \pi} N_{E N}^{1}\left(\pi_{o p}\right)-\mu \frac{\mathrm{d} N_{E N}^{I}\left(\pi_{o p}\right)}{\mathrm{d} \pi}=0 .
\end{aligned}
$$

The solution is

$$
\pi_{o p}=\frac{\phi}{\delta} \bullet(\psi \bullet \mu+\varphi)
$$

where

$$
\begin{aligned}
& \delta=(1-p)\left(a+b^{0}\right)^{2}\left(a+2 b^{1}\right)+p\left(a+b^{1}\right)^{2}\left(a+2 b^{0}\right) \\
& \phi=\left(a+b^{0}\right)\left(a+b^{1}\right) \\
& \psi=p\left(a+b^{1}\right)+(1-p)\left(a+b^{0}\right) \\
& \varphi=p(1-p)\left(b^{0}\left(a+b^{1}\right) N_{P}^{0}-b^{1}\left(a+b^{0}\right) N_{P}^{1}\right) \\
& \text { with } N_{P}^{0}=\frac{d-k^{0}}{a+b^{0}} \text { and } N_{P}^{1}=\frac{d-k^{1}}{a+b^{1}} .
\end{aligned}
$$

It can be seen that $\pi_{o p}$ is an increasing function of $\mu$.

A sufficient second-order condition for the maximization is

$$
\frac{\mathrm{d}^{2} W G}{\mathrm{~d} \pi^{2}}=-\frac{1}{(1-p)\left(a+b^{0}\right)}\left(1+\frac{b^{0}}{a+b^{0}}\right)-\frac{1}{p\left(a+b^{1}\right)}\left(1+\frac{b^{1}}{a+b^{1}}\right)<0 .
$$

Second, we will show that when three conditions are satisfied, that $\pi_{m}>\pi_{o p}$ for $\mu \epsilon$ $\left[0, \pi_{\max }\right]$ will hold. The three conditions are: (i) both prices are an increasing function of $\mu$, (ii) the coefficient of $\mu$ in $\pi_{o p}$ is greater than that in $\pi_{m}$, and (iii), given condition (i) and (ii), the horizontal coordinate of the intersection of $\pi_{m}$ and $\pi_{o p}$ is greater than $\pi_{\max }$. The proof is given below, with the assumption that $k^{0}=k^{1}=k$.

Firstly, from (10) and (A17), it can be seen that Condition (i) holds. Next, denoting the difference of the coefficients of $\mu$ in the two prices as diffcoef, then

diffcoef $=$ coefficient of $\mu$ in $\pi_{o p}$ - coefficient of $\mu$ in $\pi_{m}=\frac{\theta}{2 \delta}$,

with $\theta=a\left(a^{2}+2 a\left((1-p) b^{0}+p b^{1}\right)+(1-p) b^{0^{2}}+p b^{1^{2}}\right)$, and $\delta$ given in (17A). Obviously, diffcoef $>0$ - Condition (ii) is satisfied. Finally, before proceeding, we give the derivation of $\pi_{\max }$, which is presented in Appendix 7.A1 in Emmerink (1998). 
In state 0 , the maximal price $\pi_{\max }^{0}$ can be found by equating $N_{E N}^{0}$ and $N_{N}$. Using expression (5.a) in Section 2.2, it follows that

$\pi_{\max }^{0}=\left(N_{P}^{0}-N_{N}\right)(1-p)\left(a+b^{0}\right)$.

A similar reasoning for state 1 yields

$\pi_{\max }^{1}=\left(N_{N}-N_{P}^{1}\right) p\left(a+b^{1}\right)$.

Using the explicit formulas for $N_{P}^{0}, N_{P}^{1}$ and $N_{N}$, it can be easily shown that $\pi_{\max }^{0}=\pi_{\max }^{1}=\pi_{\max }$, which is given below:

$\pi_{\max }=\frac{p(1-p)\left(a\left(k^{1}-k^{0}\right)-b^{0}\left(d-k^{1}\right)+b^{1}\left(d-k^{0}\right)\right)}{a+p b^{1}+(1-p) b^{0}}$.

Assuming that $\pi_{m}$ and $\pi_{o p}$ insect at $(x, y)$, then

$x-\pi_{\max }=\frac{2 p(1-p)(d-k)\left(b^{1}-b^{0}\right)\left(a^{2}+a\left(b^{1}+b^{0}\right)+b^{1} b^{0}\right)}{\left(a+p b^{1}+(1-p) b^{0}\right)\left(p\left(a+b^{1}\right)^{2}+(1-p)\left(a+b^{0}\right)^{2}\right)}$,

greater than zero. Therefore, Condition (iii) holds.

\section{A.2.2 Regime under first-best road pricing}

The welfare gain from information is

$W G^{*}=T S B^{*}-\mu \bullet\left(N_{E N}^{0^{*}}-N_{E N}^{I^{*}}\right)-F C$

where $T S B^{*}$ is calculated in the same way as TSB above. The maximization problem is then

$$
\max _{\pi} W G^{*}
$$

subject to the constraints stated in (A10).

The optimal information price, denoted by $\pi_{o p}^{*}$, are found by first substituting (A12.a) and (A12.b) into (A24) to express $W G^{*}$ as a function of $\pi$. The first ordercondition is then obtained by taking derivative of $W G^{*}$ with respect to $\pi$, which is not provided here due to limited space. Only the solution is given, which is

$\pi_{o p}^{*}=\mu$.

\section{A.3 Information subsidy}

In this appendix, we will show that equal amount of a user or a producer subsidy can be implemented to arrive at the same optimal $W G$. To make the calculations simple, we express demand for information in (6) in the following way: 
$N_{E N}^{\operatorname{Inf}}(\pi)=\alpha-\beta \pi$,

where $\alpha=N_{p}^{0}-N_{p}^{1}$ represents the intercept of the demand function, whereas $\beta$ stands for the term in the parentheses in (6). The inverse form of the demand for information can be written as

$\pi\left(N_{E N}^{I n f}\right)=\frac{1}{\beta}\left(\alpha-N_{E N}^{I n f}\right)$.

Under a user subsidy, which we denote by $u s$, the monopolist's profit can be written as $(\text { profit })_{u s}^{m}=-\frac{1}{\beta}\left(N_{E N}^{I n f}\right)^{2}+\left(\frac{\alpha}{\beta}+u s-\mu\right)$.

Under a producer subsidy, the monopolist's profit function is

$(\text { profit })_{p s}^{m}=-\frac{1}{\beta}\left(N_{E N}^{I n f}\right)^{2}+\left(\frac{\alpha}{\beta}+p s-\mu\right)$.

The superscript ' $m$ ' in (A29) and (A30) represents the monopolist's pricing strategy. We use $\left(N_{E N}^{I n f}\right)_{u s}^{m}$ and $\left(N_{E N}^{I n f}\right)_{p s}^{m}$ to denote the profit maximization outputs under the user and producer subsidy respectively. Then these two equilibria can be written as

$$
\begin{aligned}
& \left(N_{E N}^{I n f}\right)_{u s}^{m}=\frac{1}{2} \alpha-\frac{1}{2} \beta \mu+\frac{1}{2} \beta u s \\
& \left(N_{E N}^{I n f}\right)_{p s}^{m}=\frac{1}{2} \alpha-\frac{1}{2} \beta \mu+\frac{1}{2} \beta p s
\end{aligned}
$$

By equating $u s$ and $p s$ in (A32), we can see that equal amount of a user or a producer subsidy leads to identical equilibria on the road. Moreover, at the optimal $W G$,

$u s_{o p}^{m}=p s_{o p}^{m}$,

where $u s_{o p}^{m}$ and $p s_{o p}^{m}$ represent the user and producer subsidy respectively that maximize $W G$.

\section{A.4 Explanation of decreasing profit in demand elasticities for mobility}

According to the experiment settings regarding the change of demand elasticities for mobility in Section (3.4), we need to write the demand function for mobility in such a way that it has a slope $a$ and crosses the point $\left(C_{N}, N_{N}\right)$. Such a demand function can be written as

$D(N)=C_{N}+a N_{N}-a N$

Using (A33), demand for information, which we denote by $N_{E N}^{\operatorname{Inf}}(\pi)$, can be expressed as 
$N_{E N}^{I n f}(\pi)^{\prime}=\frac{C_{N}+a N_{N}-k^{0}}{a+b^{0}}-\frac{C_{N}+a N_{N}-k^{1}}{a+b^{1}}-\pi \frac{p(1-p)\left(a+b^{0}\right)\left(a+b^{1}\right)}{(1-p)\left(a+b^{0}\right)+p\left(a+b^{l}\right)}$,

which is linear in $\pi$. Using $\xi$ and $\zeta$ to represent the intercept and slope of (A34), demand for information can be written as

$N_{E N}^{I n f}{ }^{\prime}(\pi)=\xi-\zeta \pi$

The inverse demand for information can be formulated as

$\pi^{\prime}\left(N_{E N}^{\operatorname{Inf}}\right)=\frac{\xi}{\zeta}-\frac{1}{\zeta} N_{E N}^{\operatorname{Inf}}$

where $\frac{\xi}{\zeta}$ is the intercept of (A36) and is equal to $\pi_{\max }{ }^{\prime}$, which can be found by equating $\pi_{\max }^{0}{ }^{\prime}$ or $\pi_{\max }^{1}{ }^{\prime}$ with $N_{N}$ (see Appendix A.2.1). As a result, $\frac{\xi}{\zeta}$ can be expressed as

$\frac{\xi}{\zeta}=\pi_{\max }{ }^{\prime}=\pi_{\max }^{0}=(1-p)\left(C_{N}-b^{0} N_{N}-k^{0}\right)$.

Obviously, $\frac{\xi}{\zeta}$ is invariant of $a$. From (A34) and (A35), we can see that $\frac{1}{\zeta}$ is increasing in $a$. The resulting monopolistic price and output are

$$
\pi_{m}^{\prime}=\frac{\xi}{2 \zeta}+\frac{\mu}{2}
$$

and

$$
N_{m}^{\prime}=\frac{\xi}{2}-\frac{\zeta}{2} \mu
$$

From the results derived above, $\pi_{m}$ in (A38) does not change with $a$. And since $\frac{\xi}{2}=\frac{\xi}{2 \zeta} / \frac{1}{\zeta}$, decreasing in $a$, and the same for $\zeta, N_{m}$ in (A39) is decreasing in $a$ as well. Combining (A38) and (A39), we can draw the conclusion that when demand for mobility becomes relatively inelastic (or the slope of the inverse demand for mobility increases), the monopolist's profit will fall. 